\title{
EFICIENCIA DE USO DE AGUA DE LA PAPA EN RESPUESTA A LA APLICACIÓN DE DIFERENTES NIVELES DE RIEGO UTILIZANDO EL RIEGO PARCIAL EN CONDICIONES DE INVERNADERO
}

\section{WATER USE EFFICIENCY OF POTATO IN RESPONSE TO APPLICATION OF DIFFERENT LEVELS OF IRRIGATION USING PARTIAL IRRIGATION IN GREEN HOUSE CONDITIONS.}

\author{
${ }^{1}$ Wendy L. Yactayo G. \& ${ }^{2}$ Miguel A. Sánchez D.
}

\begin{abstract}
Resumen
Se llevó a cabo un experimento en un invernadero de la sede central del Centro Internacional de la Papa (CIP) en Lima, cuyo objetivo fue evaluar el efecto del riego parcial (RP) sobre el crecimiento y el uso de agua de la planta de papa (Solanum tuberosum). Treintaiseis plantas de papa de la variedad UNICA fueron cultivadas individualmente en macetas. La zona radicular de las plantas sometidas al tratamiento de (RP) fue dividida en dos secciones iguales por una membrana impermeable de plástico. Las plantas de (RP) fueron regadas frecuentemente pero de manera alternada en cada mitad de la zona de raíces. A diferencia de las plantas con tratamiento (RP), las plantas con riego normal (RN), tuvieron un riego aplicado a todo el sistema radicular. El riego se determinó en función de la transpiración medida mediante el peso diario de las macetas. Los tratamientos de riego normal tuvieron cuatro niveles de reposición del agua transpirada $(100 \%, 60 \%, 45 \%$ y $30 \%)$ siendo el nivel de $100 \%$ el tratamiento control. Los tratamientos de riego parcial tuvieron tres niveles de reposición de agua $(60 \%, 45 \%$ y 30\%), porcentajes referidos al tratamiento control. La aplicación de los tratamientos se inició a los 28 días después de la siembra embolsándose las macetas para no tener pérdidas de agua por evaporación y se prolongó por 60 días. Los resultados mostraron que todos los riegos restringidos causaron una reducción significativa en la producción de biomasa seca total y la biomasa seca de tubérculos, con respecto al tratamiento control, independientemente del tipo de riego. El riego parcial, en las condiciones de invernadero del experimento, mejoró la eficiencia de uso de agua, evaluada a través de la eficiencia de transpiración en relación a la producción de biomasa total. El incremento de la eficiencia varió entre 12\% a 20\%, independientemente del sistema de riego restringido.
\end{abstract}

Palabras clave: Papa, Riego Parcial.

\begin{abstract}
Summary
An indoor experiment was conducted at CIP's headquarters in Lima, Peru, to assess the effect of Partial Irrigation (PI) method on the growth and water consumption of potato (Solanum tuberosum) plants. Thirty six potato plants (UNICA variety) were grown individually in pots. The root zone of plants subjected to partial irrigation treatment was divided into two equal sections by an impermeable plastic membrane. PI plants were watered frequently but alternately to each half of the root zone. Plants with normal irrigation (NI) had the watering applied to the entire root system (nonbisected root zone). All plants were irrigated on demand, as defined by daily transpiration determined by the weight of the pots. Treatments contrasted normal irrigation at four levels of water supply (reposition of $100 \%, 60 \%, 45 \%$ and $30 \%$ of transpired water being the $100 \%$ level the control), with PRD irrigation at three levels of water supply (60\%, $45 \%$ and $30 \%$ of transpired water refered to the control treatment). Differential irrigation schedules were initiated 28 days after planting and the experiment lasted 90 days from planting. Results showed that all restricted irrigation caused a significant reduction in total dry biomass and tuber dry biomass, compared to control treatment, regardless of the irrigation system. In this experiment, partial irrigation improved water use efficiency, as measured by the transpiration efficiency in relation to the total biomass production. The increased efficiency ranged from $12 \%$ to $20 \%$, regardless of restricted irrigation system.
\end{abstract}

Key words: Potato, Partial Irrigation.

\section{Introducción}

El riego parcial (RP) es una técnica de riego que provee agua, de manera alternada, a la mitad de la zona radicular del cultivo, causando que la parte regada disponga de humedad suficiente mientras que en la otra mitad se produce un secado progresivo hasta el momento en que se invierte el riego y la mitad del sistema radicular que estuvo previamente bien regada, entra en secado progresivo. Por un lado el desecamiento parcial de las raíces estimula la producción de ácido abscísico (ABA) el que promueve

${ }^{1}$ Ingeniero Agrícola. Asistente de Investigación Junior, Centro Internacional de la Papa. Lima, Perú. Email: w.yactayo@cgiar.org

${ }^{2}$ Profesor principal del Departamento de Recursos Hídricos. Facultad de Ingeniería Agrícola de la Universidad Nacional Agraria La Molina, Lima,

Perú. Email: msanchez@lamolina.edu.pe 
una cierta reducción de la apertura de estomas con la consiguiente disminución de la transpiración mientras que el lado del sistema radicular bien regado mantiene un estado hídrico favorable en las hojas, las que continúan fotosintetizando a tasas normales. El resultado neto es el incremento del rendimiento por unidad de agua transpirada (eficiencia de uso de agua) con respecto a un riego convencional que usa grandes cantidades de agua (Davies et al. 2002). Este trabajo es una contribución al conocimiento de la eficiencia de transpiración de la planta de papa y de su uso del agua, tema sobre el que no hay una amplia investigación, particularmente en ambientes semi-áridos como los de la costa peruana, en los que hay escasez del recurso hídrico. Este conocimiento permitirá el diseño de sistemas de riego de campo más eficientes. La técnica del RP puede ser una alternativa que necesita ser investigada y validada al nivel de campo de agricultores y para ello es necesario tener estudios sobre las respuestas, no sólo de producción, sino también las respuestas fisiológicas y morfológicas que tienen las plantas, para saber si es posible esperar resultados promisorios con el uso del RP. El ensayo en macetas al ser un sistema en el que se puede tener mayor control, proporcionará información necesaria para saber cómo responde esta variedad de papa frente al riego parcial (RP) a diferentes láminas de agua, delimitar hasta qué punto es posible incrementar la eficiencia en el uso del agua y además saber si dicha estrategia tiene alguna ventaja frente a un riego normal con restricción de agua (riego deficitario) utilizando las mismas láminas de agua de riego y así saber si se puede esperar que el (RP) aplicado a plantas bajo condiciones de campo tengan las mismas respuestas obtenidas en este experimento. El experimento se llevó a cabo en el invernadero $\mathrm{N}^{\circ} 9$ de la sede central del CIP Lima, Perú, para evaluar la aplicabilidad del riego parcial (RP) sobre el cultivo de papa variedad UNICA.

El presente trabajo tuvo como objetivo general determinar cuál es el efecto del riego parcial (RP) sobre la eficiencia de uso de agua de las plantas de papa. Siendo el objetivo especifico determinar los cambios fisiológicos y morfológicos que se presentan en la planta de papa al aplicar el riego parcial (RP).

\section{Materiales y métodos}

\section{Materiales}

El experimento fue conducido entre los meses de agosto y diciembre del año 2008 en el invernadero $\mathrm{N}^{\circ} 9$ del Centro Internacional de la Papa (CIP) en La Molina, Lima-Perú. El suelo utilizado fue el PRO-MIX. Se realizó con 36 semillas de papa. Se trabajó con la variedad UNICA. Se utilizaron 36 macetas $\mathrm{N}^{\circ} 10$ de $25 \mathrm{~cm}$ de diámetro, distribuidas en 4 mesas, 15 macetas fueron designadas para los tratamientos de (RP) y por ello fueron separadas diametralmente con plástico en dos partes iguales a una altura prudencial para que se pudieran colocar los brotes de las semillas, esta división estuvo sellada con silicona. Las macetas se llenaron con $1.2 \mathrm{~kg}$ del sustrato. Para iniciar los riegos diferenciados, es decir, los tratamientos, todas las macetas se cubrieron con plástico, dejando un agujero en el centro por donde salían los tallos de la planta, con el fin de evitar pérdidas de agua por evaporación del suelo.

Los equipos de medición utilizados fueron:

- Balanza de precisión,

- Horno o Estufa,

- Clorofilómetro SPAD.,

- Porómetro,

- Programa Compueye LSA (software para la determinación del área de una hoja),

- 1 Microvoltímetro y 2 cámaras psicrométricas (para la determinación del potencial osmótico),

- Una computadora personal Pentium IV, 3.00 GHz., y

- Programa para el análisis estadístico de datos SAS v.9 (SAS Institute, 1990).

\section{Metodología}

La siembra se realizó el 24 de septiembre del 2008. El inicio de los tratamientos se hizo cuando las plantas alcanzaron un $20 \%$ de crecimiento y las raíces ocuparon toda la maceta que fue a los 28 días. Las macetas se pesaban y se regaban diariamente. La alternancia de riego de una mitad de la zona radicular a otra, para el caso de las plantas sometidas al tratamiento de RP, se hizo cuando habían acumulado un aproximado de 900 gramos de agua transpirada, es decir, cuando la suma del agua transpirada diariamente se aproximaba a 900 gramos. De acuerdo a la pérdida de agua por transpiración que tenían las plantas control, se calculaba el porcentaje de agua que se debía aplicar a las otras plantas según el tratamiento, sin embargo, si alguna de las plantas con tratamiento de (RP) o de (RN) transpiraba menos de lo que se había calculado para ésta, se le reponía sólo lo que había transpirado individualmente, para no darle más agua de lo que había perdido por transpiración.

\section{Tratamientos}

Las macetas fueron regadas a capacidad de campo durante los primeros 27 días luego de la siembra. Se tuvieron 5 repeticiones por cada tratamiento. A los 28 días después de la siembra, las plantas fueron sometidas a 7 tratamientos de riego los cuales fueron:

(1) Riego Normal al 100\% (RN 100), en el que se aplicó el $100 \%$ de lo transpirado por la planta diariamente.

(2) Riego Normal al 60\% (RN060), en el que se aplicó el $60 \%$ de lo transpirado en promedio por las plantas de riego normal al $100 \%$.

(3) Riego Normal al 45\% (RN045), en el que se aplicó el $45 \%$ de lo transpirado en promedio por las plantas de riego normal al $100 \%$.

(4) Riego Normal al 30\% (RN030), en el que se aplicó el $30 \%$ de lo transpirado en promedio por las plantas con 
RN 100\%. En el caso de estos primeros 4 tratamientos con RN las macetas no tuvieron ninguna división física y el agua se aplicó directamente al sustrato de forma común, siendo entonces tratamientos con la técnica de riego deficitario $(\mathrm{RD})$ utilizado en estudios anteriores como ya se mencionó. Para el caso de las plantas sometidas al RP, los tratamientos fueron:

(5) Riego Parcial al 60\% (RP060) que recibió el 60\% de lo transpirado en promedio por las plantas con RN 100\%.

(6) Riego Parcial al 45\% (RP045) que recibió el 45\% de lo transpirado en promedio por las plantas con RN 100\%.

(7) Riego Parcial al 30\% (RP030), que recibió el 30\% de lo transpirado en promedio por las plantas con RN 100\%. En el caso de estos últimos 3 tratamientos las macetas tuvieron la división de plástico al centro y la aplicación del riego se hizo a un solo lado, alternándolo cada cierto tiempo. La duración de la etapa de tratamiento fue de 60 días.

\section{Evaluaciones durante el ensayo:}

\section{Medida de la porometría}

La medida de la porometría se hizo mediante un Porómetro tipo AP4 de la marca Delta T- Devices cada 5 días durante la etapa de aplicación de tratamientos. La medición se hizo cuando había mayor intensidad de brillo solar, generalmente se hizo entre las 11:30 am - 2:00 pm. Los valores se expresaron como mmoles $/ \mathrm{m}^{-2} / \mathrm{seg}^{-1}$.

\section{Medida del potencial osmótico (PO)}

La determinación del PO se hizo sobre un foliolo localizado en una hoja del tercio superior de la planta. Mediante un muestreador de discos de hoja de $0.5 \mathrm{~cm}$ de diámetro, se sacaron dos muestras por foliolo, las cuales fueron guardadas en viales identificados y llevadas a una congeladora de $-80^{\circ} \mathrm{C}$ hasta el momento de la medición. El potencial osmótico $(\mathrm{MPa})$ se midió en las cámaras psicrométricas de la marca (Wescor), modelo C-52 y fue calculado con el microvoltímetro de la marca Wescor modelo HR-33T. Se utilizó el método del punto de rocío, el cual consiste en que una vez colocada la muestra en la cámara psicrométrica, y transcurridos no menos de 30 minutos de tiempo de equilibrio de la muestra, se conecta la cámara con el microvoltimetro, corrigiendo la temperatura de la cámara, sobre un valor del microvoltimetro, siguiendo el procedimiento del manual de operación, que establece los pasos para determinar el potencial osmótico de una muestra, expresado en bar o $\mathrm{MPa}$.

\section{Medida de la clorofila}

La determinación del contenido de clorofila en las hojas se hizo mediante un medidor de clorofila instantáneo, Konica Minolta - Spad 502 cada 5 días. La medida se hizo en los tres primeros foliolos de la misma hoja marcada para la medición de porometría. Se hicieron 9 lecturas (tres por cada foliolo) y se anotó el promedio que el mismo equipo determina. Las unidades en las que se obtienen las lecturas son las unidades SPAD.

\section{Medida del contenido relativo de agua (CRA) y el área específica de la hoja $(\mathrm{AEH})$}

El contenido relativo de agua y el área específica de la hoja se determinaron sacrificando el foliolo utilizado para la determinación del potencial osmótico. Esto se realizó cada 10 días, siguiendo la metodología descrita por Liu et al., (2005). El muestreo se hizo temprano por la mañana (6:00 am - 7:00 am). Lo primero que se hizo con los foliolos obtenidos fue pesarlos en fresco (PF) utilizando una balanza analítica, luego se identificaron y sumergieron en agua destilada durante 6 horas, para luego volverlos a pesar y obtener el peso túrgido (PT). En ese momento se aprovechó la turgencia de los foliolos para escanearlos utilizando el programa CompuEye, LSA y obtener el área de cada foliolo (AF). Luego, los foliolos fueron llevados al horno durante 20 horas aproximadamente y a $100^{\circ} \mathrm{C}$ para obtener su peso seco (PS).

El CRA se obtuvo con la siguiente relación: $\mathrm{CRA}=(\mathrm{PF}-$ PS)/(PT-PS) $\left(\mathrm{g} / \mathrm{g}^{-1}\right)$.

El AEH se obtuvo con la siguiente relación: $\mathrm{AEH}=\mathrm{AF} /$ PS $\left(\mathrm{cm}^{2} \mathrm{~g}^{-1}\right)$.

\section{Luego de la cosecha}

Se pesó y se obtuvo el peso seco de la biomasa total producida por cada planta. La eficiencia de transpiración de las plantas en los diferentes tratamientos se calculó con las siguientes relaciones:

ET $($ biomasa $)=$ Peso biomasa total seca $(\mathrm{g}) /$ total agua transpirada $(\mathrm{kg})$.

ET (tubérculos) $=$ Peso seco de tubérculos $(\mathrm{g}) /$ total agua transpirada $(\mathrm{kg})$

La determinación del volumen total de agua utilizada por planta, para tener una proyección a condiciones de campo, se hizo utilizando la relación del total de agua transpirada con respecto al área de la maceta y llevándolo a las unidades correspondientes, de la siguiente forma:

Vol. $(\mathrm{mm})=(($ Total de agua transpirada $(\mathrm{g}) /$ Área de maceta $\left.\left.\left(\mathrm{m}^{2}\right)\right)^{*} 0.01\right) / 10\left(\mathrm{~m}^{3} \mathrm{ha}^{-1}\right)$

Donde: Área de maceta $=0.0509 \mathrm{~m}^{2}$

La determinación del rendimiento por planta, para tener una proyección a condiciones de campo, se hizo calculando en primer lugar el número de plantas por hectárea de la siguiente manera:

$\mathrm{N}^{\mathrm{o}}$ plantas $=\left(10000 \mathrm{~m}^{2}(1 \mathrm{ha}) /\right.$ Área de influencia de la planta $\left.\mathrm{m}^{2}\right)=40000$ plantas.

Donde: Área de influencia de la planta $=0.25 \mathrm{~m}^{2}(0.5 \mathrm{~m}$ x $0.5 \mathrm{~m}$ ).

Este valor se determinó estimando el área cuadrada que ocupaba toda la cobertura de la planta. Para ello se calculó en promedio la distancia que la planta sobresalía 
de la maceta y el valor promedio obtenido fue de 12.5 $\mathrm{cm}(0.125 \mathrm{~m})$, tomando en cuenta además el diámetro de la maceta. Finalmente el rendimiento se obtuvo con la siguiente relación:

Rendimiento $\left(\mathrm{t} \mathrm{ha}^{-1}\right)=\left(\mathrm{N}^{\mathrm{o}}\right.$ plantas $\left./ \mathrm{ha}\right) *$ Peso fresco de tubérculos $(\mathrm{g}) / 1000000$

\section{Resultados y discusión}

\section{Respuestas fisiológicas: porometría}

La conductancia estomática (medida con el Porómetro) en promedio disminuyó en el tiempo (Figura 1) una vez iniciados los tratamientos, variando en promedio desde $431 \mathrm{mmol} \mathrm{m}^{-2} \mathrm{seg}^{-1}$ (a los 8 días después de iniciado el tratamiento) hasta $136 \mathrm{mmol} \mathrm{m} \mathrm{m}^{-2} \mathrm{seg}^{-1}$ (a los 51 días después de iniciado el tratamiento). Esta disminución de la conductancia es una respuesta al estado de humedad en el suelo, el cual induce señales en la raíces que posiblemente contribuyan a un cierre estomático (Liu et al., 2005).

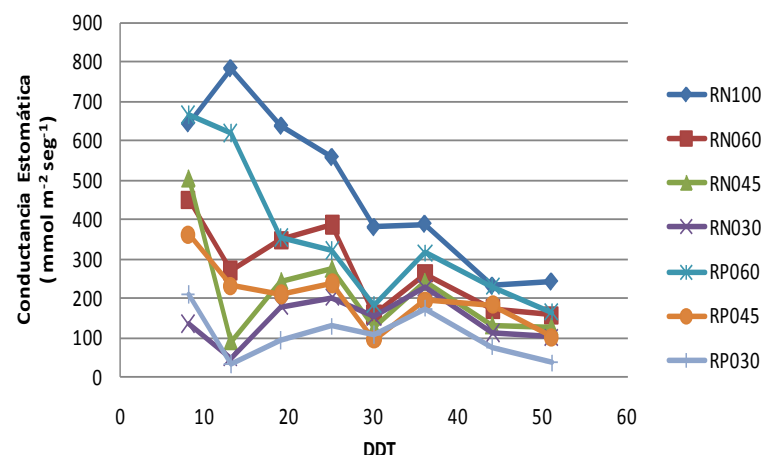

Figura 1. Conductancia estomática $\left(\mathrm{mmol} \mathrm{m}^{-2} \mathrm{seg}^{-1}\right)$.

La medición de la conductancia estomática en un estado final de la prueba, mostró que todos los tratamientos de riego difieren significativamente del tratamiento control (243 $\mathrm{mmol} \mathrm{m}^{-2} \mathrm{seg}^{-1}$ ), obteniéndose valores de un nivel menor en los tratamientos RP030 (37 mmol m $\left.\mathrm{meg}^{-1}\right)$, RN030 (102 $\mathrm{mmol} \mathrm{m}^{-2} \mathrm{seg}^{-1}$ ) y RP 045 (102 $\mathrm{mmol} \mathrm{m}^{-2} \mathrm{seg}$ $\left.{ }^{1}\right)$, los que presentan diferencias altamente significativas (Cuadro 1), expresando un mayor nivel de estrés en la planta. Lo mismo sucedió en estudios anteriores en papa, en los que en la mayoría de las mediciones, la conductancia estomática fue significativamente menor en los tratamientos con riego parcial que en el riego control (Shahnazari et al., 2007).

Tabla 1. Conductancia estomática - 50ddt.

\begin{tabular}{lccc}
\hline Tratamiento & $\begin{array}{c}\text { Conductancia } \\
\text { estomática (mmol } \\
\mathrm{m}^{-2} \mathrm{~s}^{-1}+/- \text { CME }\end{array}$ & PR & $\begin{array}{c}\text { Prueba } \\
\text { de t }\end{array}$ \\
\hline RN100 & $243+/-15$ & & \\
RN060 & $157+/-28$ & $35.4 \%$ & $0.0179^{*}$ \\
RN045 & $127+/-42$ & $48.0 \%$ & $0.0198^{*}$ \\
RN030 & $102+/-16$ & $58.0 \%$ & $0.0001^{* *}$ \\
\hline
\end{tabular}

Continuación de tabla 1

\begin{tabular}{lccc}
\hline RP060 & $165+/-21$ & $32.0 \%$ & $0.0109^{*}$ \\
RP045 & $102+/-21$ & $58.0 \%$ & $0.0002^{* *}$ \\
RP030 & $37+/-11$ & $84.0 \%$ & $0.0000^{* *}$ \\
\hline
\end{tabular}

** Altamente significativo.*Significativo. PR: Porcentaje de reducción con respecto al tratamiento control

CME: Cuadrado medio del error

\section{Potencial osmótico:}

El potencial osmótico de las mediciones realizadas se incrementa, en promedio, con el transcurso del tiempo (Figura 2), desde valores de $-1.25 \mathrm{MPa}$ (a los 9 días de iniciado el tratamiento) hasta $-1.62 \mathrm{MPa}$ (a los 41 días de iniciado el tratamiento). Este incremento puede ser explicado por la mayor acumulación de solutos (azúcares, aminoácidos, etc.) en los estadios intermedios y finales de crecimiento.

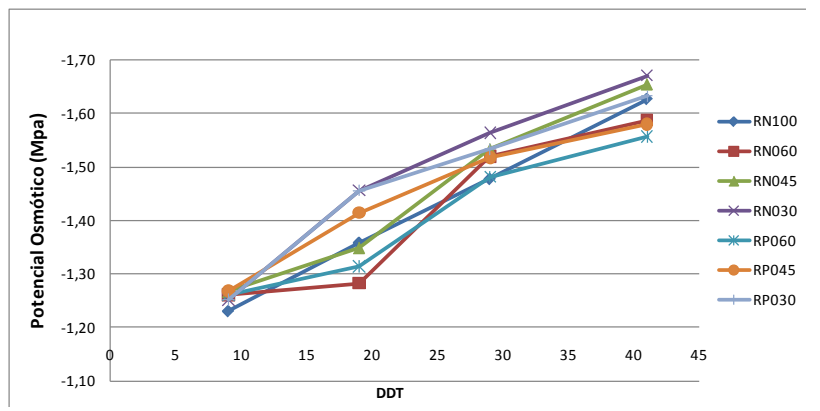

Figura 2. Potencial osmótico (Mpa).

En los tratamientos de riego no hay diferencias estadísticas significativas para el potencial osmótico medido en las fechas de evaluación (9 ddt, 19 ddt, 29 ddt y $41 \mathrm{ddt}$ ), tabla 2. Sin embargo, en la última fecha se presenta una tendencia de incremento en el valor absoluto para los tratamientos con una mayor limitación en el riego (RN030, RN045, RP030), pero no existen diferencias entre los tratamientos de riego aplicados y el riego control (RN100) para la prueba de t realizada. Esta ausencia de diferencias en el potencial osmótico puede deberse a características de la variedad, la cual quizás no incrementa el contenido de solutos en los tejidos de la célula, ante la presencia de un riego limitado.

Tabla 2. Potencial osmótico - 41ddt.

\begin{tabular}{lcccc}
\hline Tratamiento & $\begin{array}{c}\text { Potencial Osmótico } \\
(\mathrm{Mpa})+/- \text { CME }\end{array}$ & $\mathrm{CV}$ & $\begin{array}{c}\text { Prueba } \\
\text { de t }\end{array}$ \\
\cline { 1 - 2 } RN100 & $-1.63+/-0.019$ & & \\
RN060 & $-1.59+/-0.046$ & $3 \%$ & $0.4212 \mathrm{~ms}$ \\
RN045 & $-1.65+/-0.028$ & $7 \%$ & $0.4228 \mathrm{~ms}$ \\
RN030 & $-1.67+/-0.016$ & $4 \%$ & $0.1196 \mathrm{~ms}$ \\
RP060 & $-1.56+/-0.044$ & $2 \%$ & $0.1542 \mathrm{~ms}$ \\
RP045 & $-1.58+/-0.040$ & $6 \%$ & $0.2827 \mathrm{~ms}$ \\
RP030 & $-1.63+/-0.019$ & $3 \%$ & $0.827 \mathrm{~ms}$ \\
n.s.No sgniticativos CV: Coeficiente de variabilidadCEM: Cuadrado d \\
medio del error
\end{tabular}




\section{Contenido de clorofila:}

El contenido de clorofila disminuyó en el tiempo (Fig. 3). Para todas las fechas evaluadas, el tratamiento control (RN100) mantiene en promedio los menores valores de contenido de clorofila, del mismo modo fueron los tratamientos con la mayor limitación del riego (RN030 y RP030) los que alcanzaron mayores contenidos de clorofila. Las diferencias entre el tratamiento control y los demás tratamientos (prueba de t) son altamente significativas (en la última fecha de medición (56 ddt), cuadro 3. En general los contenidos de clorofila (en unidades SPAD) aumentan en plantas estresadas o que recibieron riego limitado. Este incremento puede estar asociado a un mecanismo adaptativo en la planta frente al estrés hídrico, como es el cambio en el espesor de la hoja, o también debido a la utilización limitada de nutrientes en el sustrato de la maceta por efecto del estrés hídrico o a la interrupción del crecimiento en la planta.

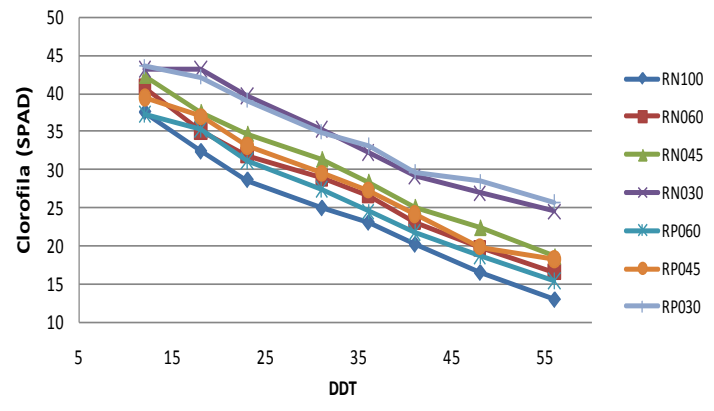

Figura 3. Contenido de clorofila (SPAD).

La presencia de clorofila en las hojas de las plantas está estrechamente relacionada con las condiciones nutricionales. El contenido de clorofila se incrementa proporcionalmente a la cantidad de nitrógeno (un importante nutriente) presente en la hoja. En el experimento, una vez iniciado los tratamientos, ya no hubo mayores aportes de fertilizantes a las macetas y posiblemente las plantas con mayor reposición de agua y mayor producción de biomasa (plantas grandes) consumieron todo el nitrógeno del substrato hasta llegar a un déficit leve de éste, mientras que las plantas con baja reposición de agua, produjeron poca biomasa (plantas pequeñas) y tuvieron suficiente nitrógeno para mantenerlo en niveles superiores en las hojas, y consecuentemente presentar mayor contenido de clorofila, lo cual no significó mayor rendimiento como se verá más adelante, sin embargo, presentaron un color de hojas más intenso.

Tabla 3. Contenido de clorofila - 56ddt.

\begin{tabular}{lcccc}
\hline Tratamiento & $\begin{array}{c}\text { Contenido de } \\
\text { clorofila (SPAD) } \\
+/- \text { CME }\end{array}$ & PI & $\begin{array}{c}\text { Prueba } \\
\text { de t }\end{array}$ \\
\cline { 2 - 3 } RN100 & $\begin{array}{c}12.9+/-0.34 \\
\text { RN060 }\end{array}$ & $16.6+/-0.059$ & $3 \%$ & $0.0003 * *$ \\
\hline
\end{tabular}

Continuación de tabla 3

\begin{tabular}{llll}
\hline RN045 & $18.6+/-0.76$ & $7 \%$ & $0.0000 * *$ \\
RN030 & $24.5+/-0.24$ & $4 \%$ & $0.0000 * *$ \\
RP060 & $15.4+/-0.43$ & $2 \%$ & $0.0012 * *$ \\
RP045 & $18.2+/-0.61$ & $6 \%$ & $0.0000 * *$ \\
RP030 & $25.7+/-0.62$ & $3 \%$ & $0.0000 * *$ \\
\hline
\end{tabular}

**Altamente significativo.* Significativo. PI: Porcentaje de incremento con respecto al tratamiento control. CME: Cuadrado medio del error

\section{Área específica de la hoja:}

Las evaluaciones para el área específica de la hoja fueron realizadas en cuatro fechas, las mismas que mostraron diferencias entre los tratamientos de riego (Figura 4). En general, se presenta una tendencia decreciente al final del desarrollo de la planta debido a que la madurez de la hoja hace que esta incremente su espesor.

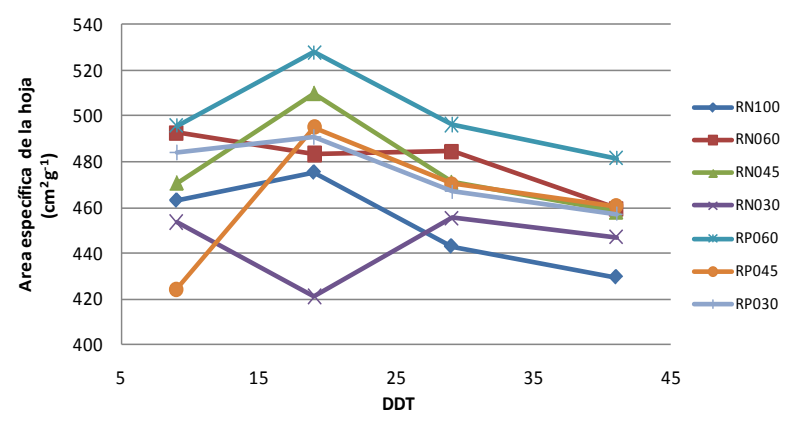

Figura 4. Área específica de la hoja $\left(\mathrm{cm}^{2} \mathrm{~g}^{-1}\right)$.

Para la comparación del área específica de la hoja en la última fecha de evaluación, en promedio de los tratamientos, no se encontraron diferencias significativas entre el tratamiento control y el tratamiento RN030, mientras que en los demás tratamientos existieron diferencias estadísticas significativas (4), siendo 481.7 $\mathrm{cm}^{2} \mathrm{~g}^{-1}$ el valor máximo obtenido con el tratamiento RP 060 y $447.1 \mathrm{~cm}^{2} \mathrm{~g}^{-1}$ el valor mínimo obtenido con el tratamiento RN 030.

Tabla 4. Área específica de la hoja - 41 ddt.

\begin{tabular}{llllll}
\hline Tratamiento & $\begin{array}{c}\text { Área específica } \\
\text { de la hoja (cm2 } \\
\text { g-1)+/- CME }\end{array}$ & PI & Prueba de t \\
\hline RN100 & $429,6 \pm 5,0$ & & & \\
RN060 & $459,5 \pm 9,0$ & $7 \%$ & 0,0138 & $*$ \\
RN045 & $458,1 \pm 5,2$ & $6 \%$ & 0,0034 & $* *$ \\
RN030 & $447,1 \pm 10,6$ & $4 \%$ & 0,1463 & ns \\
RP060 & $481,7 \pm 5,6$ & $12 \%$ & 0,0001 & $* *$ \\
RP045 & $460,7 \pm 5,3$ & $7 \%$ & 0,0021 & $* *$ \\
RP030 & $457,2 \pm 11,2$ & $6 \%$ & 0,0398 & $*$ \\
\hline
\end{tabular}

** Altamente significativo.* Significativo. n.s. No significativo PI: Porcentaje de incremento con respecto al tratamiento control CME: Cuadrado medio del error 


\section{Contenido relativo de agua}

Las evaluaciones para el contenido relativo de agua también se realizaron en cuatro fechas y se encontró que en promedio los valores disminuyeron desde $0.92 \mathrm{~g} \mathrm{~g}^{-1}$ (para la primera fecha de evaluación), hasta $0.88 \mathrm{~g} \mathrm{~g}^{-1}$ (para la última fecha de evaluación). El comportamiento de esta variable para los tratamientos RN 100, RN 060, RN 045, y RP 060, RP 045 y RP 030 tiende a ser decreciente en las tres primeras fechas y ligeramente creciente para la última fecha, sin embargo, para el tratamiento RN 030 tiende a ser decreciente para las cuatro fechas (Fig. 5).

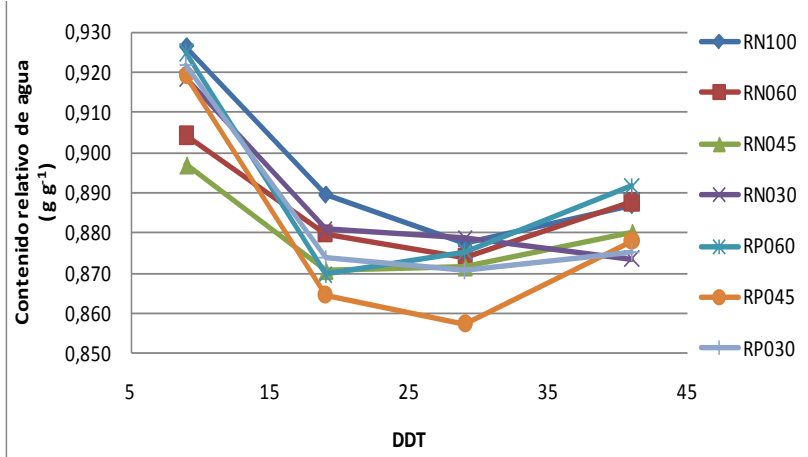

Figura 5. Contenido relativo de agua $\left(\mathrm{g} \mathrm{g}^{-1}\right)$.

En la última fecha de medición, se observa que sólo el tratamiento RN 030 presenta diferencias significativas con respecto al tratamiento control (RN 100), mientras que los otros tratamientos no presentan diferencias significativas para la prueba de $t$ (Tabla), sin embargo es el tratamiento RP 060, es el que presenta mayor contenido relativo de agua con respecto a los demás, incluyendo el tratamiento control. Posiblemente esta variedad tiene como respuesta , mantener el estado de turgencia en las hojas, que es una medida indirecta del contenido relativo de agua, lo que no sucedió en maíz, por ejemplo, donde el contenido relativo de agua si tuvo diferencias significativas en todos los tratamientos con respecto al control y entre tratamientos (Li et al., 2010)

Tabla 5. Contenido relativo de agua $-41 \mathrm{ddt}$.

\begin{tabular}{lcccccc}
\hline Tratamiento & \multicolumn{3}{c}{$\begin{array}{c}\text { Contenido relativo } \\
\text { de agua }(g g-1)+/-\end{array}$} & CV & Prueba de t \\
\hline CME & 0,887 & \pm & 0,006 & $2 \%$ & & \\
RN060 & 0,888 & \pm & 0,007 & $2 \%$ & 0,92 & ns \\
RN045 & 0,880 & \pm & 0,004 & $1 \%$ & 0,39 & ns \\
RN030 & 0,874 & \pm & 0,004 & $1 \%$ & 0,09 & $*$ \\
RP060 & 0,892 & \pm & 0,006 & $2 \%$ & 0,59 & ns \\
RP045 & 0,878 & \pm & 0,005 & $1 \%$ & 0,28 & ns \\
RP030 & 0,875 & \pm & 0,006 & $1 \%$ & 0,19 & ns \\
\hline
\end{tabular}

** Altamente significativo, * Significativo, ns. No significativo CV Coeficiente de variación CME: Cuadrado medio del error

\section{Producción de biomasa \\ Rendimiento}

El rendimiento determinado a partir del peso fresco de tubérculos (PFTu) tuvo una disminución significativa en todos los tratamientos de riego restringido con respecto al control (Tabla 6). Esto se debe posiblemente a la limitación de humedad sufrida durante toda la etapa de tratamiento, lo que puede haber afectado el llenado de los tubérculos. Este resultado no coincide con lo encontrado por Shahnazari et al (2007), quienes a la cosecha final obtuvieron mayor rendimiento en el tratamiento con RP que en tratamiento control. Mientras que Liu et al (2006b), no encuentra diferencias significativas entre el tratamiento de RPy el tratamiento control. La comparación entre los tratamientos de riego muestra que los mayores rendimientos se dieron en el tratamiento RP060, seguido del tratamiento RN060 los que fueron significativamente superiores a los de los otros tratamientos de riego.

Tabla 6. Rendimiento.

\begin{tabular}{lcccccc}
\hline Tratamientos & \multicolumn{2}{c}{$\begin{array}{c}\text { Rendimiento (t PFTu } \\
\text { ha }{ }^{-1} \text { ) }+ \text { - CME }\end{array}$} & PR & CV \\
\hline RN100 & 22 & \pm & 0.60 & $\mathrm{a}$ & & $6.6 \%$ \\
RN060 & 15 & \pm & 0.42 & $\mathrm{~b}$ & $32 \%$ & $6.2 \%$ \\
RN045 & 13 & \pm & 0.30 & $\mathrm{c}$ & $43 \%$ & $5.4 \%$ \\
RN030 & 8 & \pm & 0.08 & $\mathrm{~d}$ & $63 \%$ & $2.0 \%$ \\
RP060 & 16 & \pm & 0.14 & $\mathrm{~b}$ & $27 \%$ & $1.9 \%$ \\
RP045 & 12 & \pm & 0.74 & $\mathrm{c}$ & $46 \%$ & $13.6 \%$ \\
RP030 & 9 & \pm & 0.13 & $\mathrm{~d}$ & $61 \%$ & $3.4 \%$ \\
\hline Análisis de varianza & & & \\
\hline
\end{tabular}

Valores con la misma letra no tienen diferencias significativas para la prueba de Tukey ** PR: Porcentaje de reducción con respecto al tratamiento control, $\mathrm{CV}$ : Coeficiente de variabilidad CME: Cuadrado medio del error

En ambas técnicas de riego (RP y RN), vemos que a medida que hay mayor limitación de agua, hay también un disminución en el peso de tubérculos frescos y en consecuencia en el rendimiento, siendo ésta disminución menor en el riego parcial (RP), donde para iguales niveles de riego, este supera al riego deficitario (RP), salvo en los tratamientos RN045 Y RP045.

\section{Peso seco de tubérculos}

El peso seco de tubérculos para los tratamientos de riego disminuyó significativamente, con respecto al riego control (Tabla 7). Similares cantidades de agua aplicada no tuvieron diferencias significativas en la producción de materia seca de tubérculo, independientemente a la forma de aplicación del riego. Los niveles de riego RP060 y RN060 fueron superiores a los demás, pero sin diferencias significativas entre ambos. Liu et al., (2006a) obtuvieron mayor rendimiento de peso seco de tubérculos en el 
tratamiento de riego deficitario (en nuestro caso riego normal) con un nivel de reposición de agua de $50 \%$, que en los tratamientos de RP (también a 50\% de reposición) o control ( $100 \%$ de reposición).

Tabla 7. Peso seco de tubérculos (PSTu).

\begin{tabular}{|c|c|c|c|c|c|c|}
\hline \multirow{2}{*}{$\begin{array}{l}\text { Tratamientos } \\
\text { RN100 }\end{array}$} & \multicolumn{4}{|c|}{$\begin{array}{c}\text { Peso seco de } \\
\text { tubérculos }\left(\mathrm{g} \mathrm{pta}^{-1}\right) \\
+/-\mathrm{CME}\end{array}$} & \multirow[t]{2}{*}{ PR } & \multirow{2}{*}{$\frac{\mathrm{CV}}{7,6 \%}$} \\
\hline & 114 & \pm & 3,52 & $\mathrm{a}$ & & \\
\hline RN060 & 78 & \pm & 1,33 & $\mathrm{~b}$ & $32 \%$ & $3,8 \%$ \\
\hline RN045 & 66 & \pm & 1,71 & $\mathrm{c}$ & $42 \%$ & $5,8 \%$ \\
\hline RN030 & 49 & \pm & 1,28 & d & $57 \%$ & $5,8 \%$ \\
\hline RP060 & 82 & \pm & 1,42 & $\mathrm{~b}$ & $28 \%$ & $3,9 \%$ \\
\hline RP045 & 66 & \pm & 0,97 & $\mathrm{c}$ & $42 \%$ & $3,3 \%$ \\
\hline RP030 & 44 & \pm & 0,90 & $\mathrm{~d}$ & $61 \%$ & $4,5 \%$ \\
\hline \multicolumn{7}{|c|}{ Análisis de varianza } \\
\hline
\end{tabular}

Tratamientos

Valores con la misma letra no tienen diferencias significativas para la prueba de Tukey

** Altamente significativo, * Significativo, n.s. No significativo. PR: Porcentaje de reducción con respecto al tratamiento control. CV: Coeficiente de variabilidad. CME: Cuadrado medio del error

\section{Peso seco de biomasa total}

El peso seco de la biomasa total difiere significativamente en los tratamientos de riego respecto al control. Se observó que la reducción del nivel de reposición de humedad causó una reducción significativa de la biomasa total, independientemente del tipo de riego (Tabla 8). Estos resultados son similares a los encontrados en papa por Liu et al., 2006 y en tomate por Zegbe et al., 2006. Por otro lado, para el tratamiento RP060, la biomasa total producida fue mayor que en el riego normal.

Tabla 8. Peso seco de biomasa total.

\begin{tabular}{lcccccc}
\hline Tratamientos & \multicolumn{1}{c}{$\begin{array}{c}\text { Peso seco de biomasa } \\
\left(\text { g planta }^{-1}\right)\end{array}$} & PR & CV CME & PV \\
\hline RN100 & 147 & \pm & 4,6 & a & & $7,7 \%$ \\
RN060 & 105 & \pm & 1,9 & bc & $29 \%$ & $4,0 \%$ \\
RN045 & 92 & \pm & 2,3 & cd & $37 \%$ & $5,7 \%$ \\
RN030 & 75 & \pm & 2,2 & e & $49 \%$ & $6,4 \%$ \\
RP060 & 112 & \pm & 2,0 & b & $24 \%$ & $3,9 \%$ \\
RP045 & 94 & \pm & 2,3 & cd & $36 \%$ & $5,5 \%$ \\
RP030 & 72 & \pm & 1,7 & e & $51 \%$ & $5,3 \%$ \\
\hline \multicolumn{7}{c}{ Análisis de varianza } \\
\hline Tratamientos
\end{tabular}

Valores con la misma letra no tienen diferencias significativas para la prueba de Tukey

** Altamente significativo, * Significativo, n.s. No significativo PR: Porcentaje de reducción con respecto al tratamiento control. CV: Coeficiente de variabilidad CME: Cuadrado medio del error

\section{Volúmen total de agua transpirada}

El efecto de los tratamientos de riego sobre la variable volumen total de agua transpirada fue altamente significativo (Tabla 9). La comparación de medias de los tratamientos de riego mostró diferencias significativas entre los diferentes niveles de reposición, sin embargo no hubo diferencias entre iguales niveles de reposición de agua independientemente del método de riego (RN060 y RP060; RN045 y RP045; RN030 y RP030). Por otro lado, el volumen de agua total transpirada disminuyó en la medida en que se redujo el nivel de reposición de agua en el riego. Esta respuesta para el volumen total de agua transpirada frente a diferentes niveles de reposición de agua tiene una causa directa debido a que la planta utiliza el agua que tiene disponible.

Tabla 9. Volumen total de agua transpirada.

\begin{tabular}{lcccccc}
\hline \multirow{8}{*}{ Tratamientos } & \multicolumn{7}{c}{ Volumen de agua } \\
& 480 & \pm & 11 & $\mathrm{a}$ & & $5.8 \%$ \\
\hline RN100 & 306 & \pm & 3 & $\mathrm{~b}$ & $36 \%$ & $2.1 \%$ \\
RN060 & 253 & \pm & 4 & $\mathrm{c}$ & $47 \%$ & $3.2 \%$ \\
RN045 & 206 & \pm & 5 & $\mathrm{~d}$ & $57 \%$ & $5.4 \%$ \\
RN030 & 317 & \pm & 1 & $\mathrm{~b}$ & $34 \%$ & $0.9 \%$ \\
RP060 & 255 & \pm & 7 & $\mathrm{c}$ & $47 \%$ & $6.0 \%$ \\
RP045 & 202 & \pm & 6 & $\mathrm{~d}$ & $58 \%$ & $6.2 \%$ \\
RP030 & Análisis de varianza \\
\hline \multicolumn{7}{c}{$* *$} \\
\hline Tratamientos
\end{tabular}

Valores con la misma letra no tienen diferencias significativas para la prueba de Tukey ** Altamente significativo, * Significativo, n.s. No significativo. PR: Porcentaje de reducción con respecto al tratamiento control. CV: Coeficiente de variabilidad. CME: Cuadrado medio del error

\section{Eficiencia de transpiración de biomasa}

El efecto de los tratamientos de riego sobre la eficiencia de transpiración relativa a la biomasa total fue significativo. La eficiencia de transpiración promedio de los tratamientos de riego tuvo diferencias significativas con respecto al control (RN100), incrementándose cuando se presenta una limitación del riego (Figura 6). Esta mejora en la eficiencia de uso de agua coincide con los resultados encontrados en el cultivo de papa por Liu et al., (2006a), Lui et al., (2006b), Shahnazari et al., (2007), Ahmadi et al., (2010). La división física de la zona radicular en las macetas (RP) no causó diferencias estadísticas significativas en la eficiencia de transpiración para iguales niveles de reposición (Tabla 10), con respecto a las macetas sin división (RN). Este comportamiento similar en los tratamientos RN y RP puede deberse a que en el RN la cantidad de agua aplicada en cada nivel de reposición, solo tuvo una influencia en un sector del volumen de la maceta, es decir, las plantas que recibieron menos del $100 \%$ de reposición del agua transpirada sin tener división de raíces también estuvieron a un tipo de riego parcial por que al recibir menor cantidad de agua puede que parte de la raíz no haya tenido contacto con la humedad, es decir, no podemos asumir que el riego fue homogéneo en toda la maceta. Estos resultados coinciden con Liu at al., (2009a) y Sadras (2009). 
Tabla 10. Eficiencia de transpiración de biomasa.

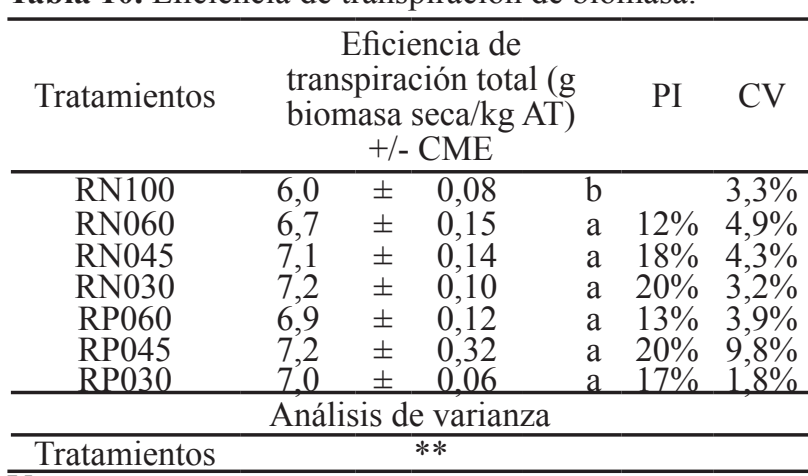

Valores con la misma letra no tienen diferencias significativas para la prueba de Tukey ** Altamente significativo, * Significativo, n.s. No significativo PI: Porcentaje de incremento con respecto al tratamiento control CV: Coeficiente de variabilidad CME: Cuadrado medio del error

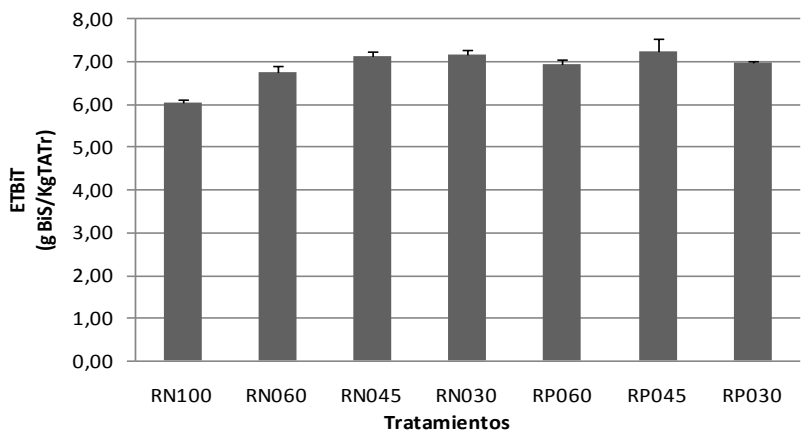

Figura 6. Eficiencia de transpiración de biomasa total $(\mathrm{g}$ $\left.\mathrm{Kg}^{-1}\right)$.

\section{Eficiencia de transpiración de tubérculo}

El análisis de variancia para la eficiencia de transpiración relativa a tubérculos resultó altamente significativa en los tratamientos de riego, con respecto al tratamiento control .En la comparación de medias para los tratamientos de riego, encontramos la mayor eficiencia de transpiración relativa a tubérculos en RN045, RP060, RP045 y RN060. En tanto la menor eficiencia se observó en el tratamiento RP030 (Tabla 11 y Figura 7) Esta respuesta de la eficiencia de uso de agua mejora cuando los niveles limitantes de riego son leves o intermedios, mientras que si estas limitaciones son severas (RN030 y RP030), la acumulación de fotosintatos en los tubérculos puede verse afectada.

Tabla 11. Eficiencia de transpiración de tubérculo.

\begin{tabular}{cccccc}
\hline Tratamientos & $\begin{array}{c}\text { Eficiencia transpiración } \\
\text { de tubérculo (g PSTU/kg } \\
\text { TAA) +/-CME }\end{array}$ & PI & CV \\
\hline RN100 & 4,67 & $\pm 0,06$ & $\mathrm{~b}$ & & $3,0 \%$ \\
RN060 & 5,01 & $\pm 0,09$ & a & $7 \%$ & $4,1 \%$ \\
RN045 & 5,15 & $\pm 0,14$ & a & $10 \%$ & $6,2 \%$ \\
RN030 & 4,68 & $\pm 0,06$ & $\mathrm{~b}$ & $0.2 \%$ & $2,9 \%$ \\
RP060 & 5,09 & $\pm 0,08$ & a & $9 \%$ & $3,5 \%$ \\
RP045 & 5,08 & $\pm 0,16$ & a & $9 \%$ & $7,2 \%$ \\
RP030 & 4,31 & $\pm 0,10$ & c & $-8 \%$ & $5,3 \%$ \\
\hline \multicolumn{7}{c}{ Análisis de varianza } \\
\hline Tratamientos
\end{tabular}

Valores con la misma letra no tienen diferencias significativas para la prueba de Tukey ** Altamente significativo, *Significativo, n.s. No significativo PI: Porcentaje de incremento con respecto al tratamiento control CV: Coeficiente de variabilidad CME: Cuadrado medio del error

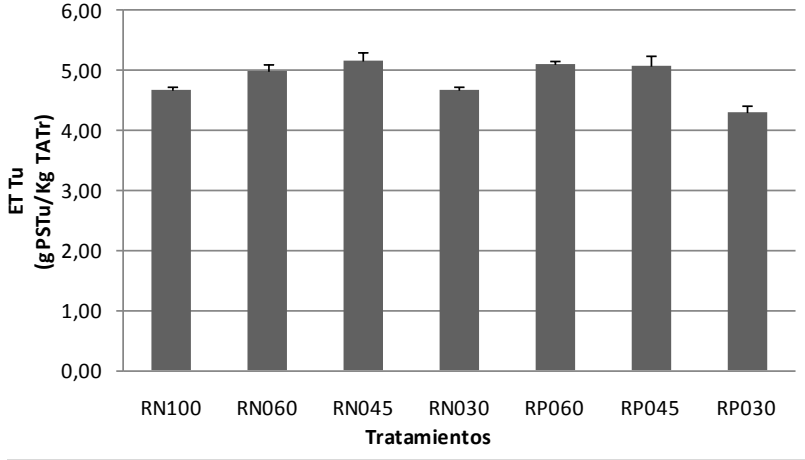

Figura 7. Eficiencia de transpiración de tubérculos (gPSTu/KgTATr).

\section{Conclusiones}

- Elriego parcial, evaluado en condiciones de invernadero, mejoró la eficiencia de uso de agua, estimada a través de la eficiencia de transpiración relativa a la biomasa total. Esta eficiencia se incrementó desde un $12 \%$ hasta un $20 \%$, en comparación con la eficiencia del riego normal.

- La eficiencia de transpiración relativa a la biomasa de tubérculo mejoró entre $7 \%$ a $10 \%$ por efecto del riego parcial, pero solo en los niveles de reposición de $60 \%$ y $45 \%$ de la transpiración diaria, con respecto al riego control.

- El riego parcial (RP) no tuvo una ventaja en la eficiencia de transpiración relativa a biomasa total y de tubérculo, con respecto al riego normal $(\mathrm{RN})$, para iguales niveles de reposición de agua.

- La conductancia estomática se redujo por efecto del riego parcial, hasta un $85 \%$ como respuesta al estado de humedad del sustrato por la reducción en el nivel de reposición de agua.

- El contenido de clorofila medida con el SPAD -502 Minolta disminuyó en el tiempo para las evaluaciones realizadas, siendo los riegos con limitados niveles de reposición de agua los que tienen los mayores valores de clorofila, hasta un $99 \%$ más con respecto al control.

- El área específica de la hoja se incrementa hasta en un $12 \%$ por efecto del riego parcial en la evaluación final (41 ddt). Mientras que el contenido relativo de agua no presenta un efecto debido al riego parcial.

- El rendimiento fue afectado por el riego parcial, habiendo una reducción hasta de 61\%. En tanto, el volumen total de agua transpirada se vio afectado disminuyendo hasta un 58\%. El peso seco de tubérculos también se redujo hasta un $57 \%$. Por otro lado, el número de tubérculos no se vio afectado por el riego parcial.

- El peso seco de la biomasa total fue afectado por el riego parcial, habiéndose reducido desde un $24 \%$ hasta un $51 \%$. 


\section{Literatura citada}

Davies, W. J;. Wilkinson, S. and Loveys, B. 2002.

Stomatal control by chemical signaling and the

exploitation of this mechanism to increase water use efficiency in agriculture. New Phytol 153: 449-460.

Fageria, N. K.; Baligar, V. C. y Clark, R. B. 2006. Physiology of Crop production. Primera edición. 153 $175 \mathrm{p}$.

Jovanovic, Z.; Stikic, R.; Vucelic, B.; Paukovic, M.; Brocic, Z.; Matovic, G.; Rovcanin, S. and Mojevic, M. 2010. Partial root-zone drying increases WUE, $N$ and antioxidant content in field potatoes. European Journal of Agronomy 33: 124-131.

Kang, S., and Zhang, J. 2004. Controlled alternate partial root-zone irrigation: its physiological consequences and impact on water use efficiency. J. Exp. Bot. 55, 2437-2446.

Kirda, C.; Topcu, S.; Kaman, H.; Ulger, A. C.; Yazici, A.; Cetin, M. and Derici, M. R. 2005. Grain yield and $\mathrm{N}$-fertilizer recovery of maize under deficit irrigation. Field Crops Res. 93, 132-141.

Li, F.; Wei, C.; Zhang, F.; Zhang, J.; Nong; M. and Kang; S. 2010. Water-use efficiency and physiological responses of maize under partial root-zone irrigation. Agricultural Water Management 97:1156-1164.

Liu, F.; Shahnazari, A.; Andersen, M. N.; Jacobsen, S. E. and Jensen, C. R. 2006. Physiological responses of potato (solanum tuberosum L.) to partial root-zone drying: ABA signaling, leaf gas exchange and water use efficiency. Dinamarca. Journal of Experimental Botany. 3727-3735.

Sadras, V. O. 2009. Does partial root-zone drying improve irrigation water productivity in the field? A meta-analysis. Irrig Sci. 27: 183-190.

Schachtman, D. P. and Goodger, J. Q. 2008. Chemical root to shoot signaling under drought. Trends in Plant Science. 13: Nº.

Shahnazari, A.; Liu, F; Andersen, M. N.; Jacobsen, S.E. and Jensen, C. R. 2007. Effects of partial root-zone drying on yield, tuber size and water use efficiency in potato under field conditions. Field Crops Research 100: 117-124.

Tanner, C. B. 1981. Transpiration efficiency of potato. Agronomy Journal. Vol. 73: 59-64.

Wang, Y.; Liu, F.; Andersen, M. N. and Jensen, C. R. 2010. Improved plant nitrogen nutrition contributes to higher water use efficiency in tomatoes under alternate partial root-zone irrigation. Functional Plant Biology. 37,175-182.

Zegbe, J.; Hossein, M. B. y Clotier, E. 2006. Respuesta del tomate para proceso al riego parcial de la raíz. México. Terra Latinoamericana 25: 61-67. 